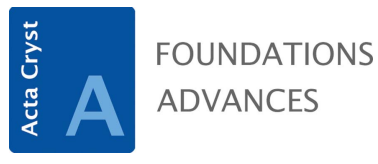

ISSN 2053-2733

\section{Mathematical Stereochemistry. By Shinsaku Fujita. De Gruyter, 2015. Pp. xviii + 437. Price EUR 129.95, USD 182.00, GBP 97.99. ISBN 978-3-11-036669-3.}

\author{
Gregory Chirikjian*
}

Johns Hopkins University, Mechanical Engineering, 223 Latrobe Hall, 3400 N. Charles Street, Baltimore MD 21218, USA. *Correspondence e-mail: gregc@jhu.edu

The author of this book, Professor Shinsaku Fujita, has had a long and productive research career as a chemist, both in industry and in academic research laboratories. This is his fourth book, which builds on the theory developed in the others while minimizing overlap.

The author states that the purpose of this book is to disambiguate vocabulary terms used in stereochemistry by introducing more precise and unambiguous group-theoretic descriptions. This book can be thought of as an application manual of sorts for the abstract methodologies established in his previous books by illustrating the theory with specific examples. In addition to the many examples presented in the book, there are links to online resources.

The core methodology used in the book is that developed in the author's previous book Symmetry and Combinatorial Enumeration in Chemistry. In particular, if $G=\{g 1, \ldots\}$ is a finite group and $H$ is a subgroup, then, by Lagrange's theorem, the right coset space $\{H g 1, \ldots\}$ has $r=|G| /|H|$ elements. The author constructs and uses this fact to build permutation representations of $G$ in the symmetric group on $r$ symbols. $\mathrm{He}$ denotes the right coset space as $G / H$ and the resulting permutation representation as $G(/ H)$ and does many calculations involving subduced representations. Unless one refers back to his previous books, it would be very difficult to understand this. [In the broader literature the notation $G / H$ is reserved for left coset spaces and right coset spaces are denoted as $H \backslash G$, and it appears that $G(/ H)$ is the author's own notation for permutation representations of $G$.]

When taken as a whole, Professor Fujita's books form a coherent body of work with minimal overlap. On the one hand, this makes each book reasonable in length, but the drawback is that Mathematical Stereochemistry is by no means self-contained. Another issue is that the author makes little effort in this book to connect directly to other works that focus on the application of finite groups to molecular symmetry. Though there are many references to Professor Fujita's own work, there are few direct references to the work of others in this book. This is not the case in some of his other books. Noticeably absent here are references to S. L. Antman's 1977 book Induced Representations in Crystals and Molecules and G. S. Ezra's 1982 book Symmetry Properties of Molecules.

In short, for quantitative stereochemists who have mastered Polya and Read's Combinatorial Enumeration of Groups, Graphs, and Chemical Compounds and have read Professor Fujita's previous books, the current book and associated online resources will be useful as a way to visualize many concrete examples of abstract ideas put into practice. 